\title{
Magnetic and diffusive nature of $\mathrm{LiFePO}_{4}$ investigated by muon spin rotation and relaxation
}

\author{
Jun Sugiyama, ${ }^{1, *}$ Hiroshi Nozaki, ${ }^{1}$ Masashi Harada, ${ }^{1}$ Kazuya Kamazawa, ${ }^{1, \dagger}$ Oren Ofer, ${ }^{2}$ Martin Månsson, ${ }^{3,4}$ Jess H. Brewer, ${ }^{2,5}$ \\ Eduardo J. Ansaldo, ${ }^{2}$ Kim H. Chow, ${ }^{6}$ Yutaka Ikedo, ${ }^{7}$ Yasuhiro Miyake, ${ }^{7}$ Kazuki Ohishi,,${ }^{8, \dagger}$ Isao Watanabe, ${ }^{8}$ \\ Genki Kobayashi, ${ }^{9, \ddagger}$ and Ryoji Kanno ${ }^{9}$ \\ ${ }^{1}$ Toyota Central Research and Development Laboratories Inc., Nagakute, Aichi 480-1192, Japan \\ ${ }^{2}$ TRIUMF, 4004 Wesbrook Mall, Vancouver, BC, V6T 2A3 Canada \\ ${ }^{3}$ Laboratory for Solid State Physics, ETH Zürich, CH-8093 Zürich, Switzerland \\ ${ }^{4}$ Laboratory for Neutron Scattering, ETH Zürich and Paul Scherrer Institut, CH-5232 Villigen PSI, Switzerland \\ ${ }^{5}$ Department of Physics and Astronomy, University of British Columbia, Vancouver, BC, V6T 1Z1 Canada \\ ${ }^{6}$ Department of Physics, University of Alberta, Edmonton, AB, T6G $2 G 7$ Canada \\ ${ }^{7}$ Muon Science Laboratory, Institute of Materials Structure Science, KEK, 1-1 Oho, Tsukuba, Ibaraki 305-0801, Japan \\ ${ }^{8}$ Advanced Meson Science Laboratory, RIKEN, 2-1 Hirosawa, Wako, Saitama 351-0198, Japan \\ ${ }^{9}$ Department of Electronic Chemistry, Interdisciplinary Graduate School of Science and Engineering, \\ Tokyo Institute of Technology, Yokohama 226-8503, Japan \\ (Received 15 April 2011; revised manuscript received 26 June 2011; published 9 August 2011)
}

\begin{abstract}
In order to elucidate the magnetism and $\mathrm{Li}$ diffusion in $\mathrm{LiFePO}_{4}$, we have measured muon-spin rotation and relaxation $\left(\mu^{+} \mathrm{SR}\right)$ spectra for the polycrystalline $\mathrm{LiFePO}_{4}$ sample in the temperature range between 1.8 and $500 \mathrm{~K}$. Below $T_{\mathrm{N}} \sim 52 \mathrm{~K}$, two oscillatory signals together with one fast relaxation signal were clearly found in the zero-field (ZF) $\mu^{+}$SR spectrum. The three signals are reasonably explained using an antiferromagnetic (AF) spin structure proposed by neutron measurements, because electrostatic potential calculations suggests multiple different muon sites in the $\mathrm{LiFePO}_{4}$ lattice. However, the AF ordered moment estimated from $\mu^{+} \mathrm{SR}$ was about $3 / 4$ of that reported by neutron, probably due to a different time window between the two techniques. In the paramagnetic state, ZF and longitudinal-field (LF) $\mu^{+}$SR spectra exhibited a dynamic nuclear field relaxation. From the temperature dependence of the field fluctuation rate, a diffusion coefficient of $\mathrm{Li}^{+}$ions $\left(D_{\mathrm{Li}}\right)$ at $300 \mathrm{~K}$ was estimated about $3.6 \times 10^{-10} \mathrm{~cm}^{2} / \mathrm{s}$, assuming that diffusing $\mathrm{Li}^{+}$ions jump between the regular site and interstitial sites.
\end{abstract}

DOI: 10.1103/PhysRevB.84.054430

PACS number(s): 76.75.+i, 66.30.H-, 82.47.Aa, 82.56.Lz

\section{INTRODUCTION}

Although diffusion of $\mathrm{Li}^{+}$ions in solids is the basic principle behind the operation of $\mathrm{Li}$-ion batteries, ${ }^{1,2}$ a reliable diffusion coefficient of $\mathrm{Li}^{+}$ions $\left(D_{\mathrm{Li}}\right)$ has not been determined for positive electrode materials. This is because the most common technique for measuring $D_{\mathrm{Li}}$, i.e., ${ }^{7} \mathrm{Li}-\mathrm{NMR}$, is known to be not suitable for materials that contain magnetic ions, due to the magnetic contribution to spin-lattice relaxation processes. ${ }^{3,4}$ In fact, $D_{\mathrm{Li}}$ estimated by ${ }^{7} \mathrm{Li}-\mathrm{NMR}$ for $\mathrm{LiCoO}_{2}$ and $\mathrm{LiNiO}_{2},{ }^{5}$ which are currently used in commercial batteries, is reported to be three or four orders of magnitude smaller than the $D_{\text {Li }}$ predicted by first-principles calculations. ${ }^{6}$ On the contrary, muons do not feel fluctuating magnetic moments at high $T$, but instead sense the change in nuclear dipole field due to Li diffusion. Even if magnetic moments still affect the muon-spin depolarization rate, such an effect is, in principle, distinguishable from that of nuclear dipole fields. In particular, a weak longitudinal field can be applied that decouples the magnetic and nuclear dipole interactions. ${ }^{7,8} \mathrm{We}$ have therefore initiated a systematic study of $D_{\mathrm{Li}}$ for battery materials by means of a muon-spin rotation and relaxation $\left(\mu^{+}\right.$SR) technique. ${ }^{9,10}$

In $\mathrm{Li}_{x} \mathrm{CoO}_{2}$, as $T$ increases from $50 \mathrm{~K}$, the field fluctuation rate $(v)$ is initially constant (eventually 0 ), but starts to increase above $160 \mathrm{~K},{ }^{10}$ around which the ${ }^{7} \mathrm{Li}$-NMR linewidth suddenly decreases due to a motional narrowing. ${ }^{11}$ Furthermore, the $T$ dependence of $v$ was well explained by a thermal activation process in the $T$ range between 160 and $250 \mathrm{~K}$. Assuming a random-walk jump of the $\mathrm{Li}^{+}$ions between the neighboring sites, $D_{\mathrm{Li}}(300 \mathrm{~K})$ for $\mathrm{Li}_{0.73} \mathrm{CoO}_{2}$ is estimated as about $6.2 \times 10^{-10} \mathrm{~cm}^{2} / \mathrm{s},{ }^{10}$ which is comparable to the prediction from first-principles calculations. ${ }^{6}$ Here, electrostatic potential calculations predict that muons locate not at the vacant $\mathrm{Li}$ site but in the vicinity of $\mathrm{O}^{2-}$ ions so as to make a stable $\mu^{+}-\mathrm{O}$ bond. ${ }^{10}$ As a result, muons sense the Li diffusion in the $\mathrm{Li}_{0.73} \mathrm{CoO}_{2}$ lattice, although the mass of $\mathrm{Li}^{+}$is heavier by 63 times than the $\mu^{+}$. For $\mathrm{LiNiO}_{2}, \mu^{+} \mathrm{SR}$ is also found to provide reasonable $D_{\mathrm{Li}}{ }^{12}$ Furthermore, for $\mathrm{LiCrO}_{2}$, which crystalizes in the same structure as $\mathrm{LiCoO}_{2}$ and $\mathrm{LiNiO}_{2}$ with similar lattice parameters, the zero-field (ZF) spectrum showed a typical static Kubo-Toyabe behavior even at $500 \mathrm{~K}$, indicating that muons are bound to the $\mathrm{O}^{2-}$ ions at least until $500 \mathrm{~K}$. Since $\mathrm{Li}$ ions are not removed from $\mathrm{LiCrO}_{2}$ by an electrochemical reaction, such a static nature is very consistent with its electrochemical properties. ${ }^{13-17}$

Following upon the $\mu^{+}$SR studies of the layered transitionmetal dioxides, we have extended the research to phosphoolivines, namely, $\mathrm{LiFePO}_{4}$ and related compounds, ${ }^{18}$ since the phospho-olivines have numerous advantages over layered transition-metal dioxides as practical battery materials. ${ }^{19,20}$ That is, besides its high capacity (approximately $170 \mathrm{mAh} / \mathrm{g}$ ), $\mathrm{LiFePO}_{4}$ shows high stability during lithium extraction/insertion and does not deteriorate when used at moderately high temperatures. Here, the $\mathrm{LiFePO}_{4}$ olivine structure consists of a distorted hexagonal close-packed (hcp) framework containing $\mathrm{Li}$ and $\mathrm{Fe}$ in octahedral sites and $\mathrm{P}$ in tetrahedral 
sites (see the inset of Fig. 1). ${ }^{21}$ It is an orthorhombic structure, space group Pnma, with lattice parameters of $a=10.3290 \AA, b=6.0065 \AA$, and $c=4.6908 \AA .^{22,23}$ During lithium extraction/insertion reaction, the $\mathrm{Li}^{+}$ions are removed topotactically while maintaining the topology of the $\mathrm{FePO}_{4}$ framework. The $\mathrm{Li}^{+}$ions were found to diffuse along the $b$ direction by neutron-diffraction measurements on a Li-deficient sample at high- $T .{ }^{24}$ However, the dynamic nature of the $\mathrm{Li}^{+}$ions is still not clarified, despite the considerable effort dedicated to fabricating Li-ion batteries using $\mathrm{LiFePO}_{4}$ as a positive electrode material. ${ }^{19}$

This is because $\mathrm{LiFePO}_{4}$ is a Curie-Weiss paramagnet down to its antiferromagnetic transition temperature $\left(T_{\mathrm{N}}=\right.$ $52 \mathrm{~K}) .^{20,21}$ Furthermore, since the electron configuration of the $\mathrm{Fe}^{2+}$ ion is $t_{2 g}^{4} e_{g}^{2}$ with $S=2$, large Fe moments affect the spin-lattice relaxation rate $\left(1 / T_{1}\right)$ of the Li-NMR signal even at ambient temperature, resulting in difficulty to observe $\mathrm{Li}$ diffusion by Li-NMR. In fact, the $1 / T_{1}(T)$ curve for $\mathrm{LiFePO}_{4}$ obtained by Li-NMR was reported to be almost $T$ independent above $51 \mathrm{~K}$ due to the effect of the paramagnetic Fe ions. ${ }^{25}$ Hence it is obviously not possible to reliably estimate $D_{\mathrm{Li}}$ by $\mathrm{NMR}$, as in the case for $\mathrm{Li}_{x} \mathrm{CoO}_{2}$. On the other hand, a recent Mössbauer study showed that $D_{\mathrm{Li}}$ of $\mathrm{LiFePO}_{4}$ ranges between $8 \times 10^{-13}$ and $3 \times 10^{-11} \mathrm{~cm}^{2} / \mathrm{s}$ at ambient temperature. ${ }^{26}$ However, first-principles calculations predicted that $D_{\mathrm{Li}} \sim 10^{-8} \mathrm{~cm}^{2} / \mathrm{s}$ for $\mathrm{Li}_{7 / 8} \mathrm{FePO}_{4}{ }^{27}$ In contrast to $D_{\mathrm{Li}}$, the chemical diffusion coefficient $\left(\tilde{D}_{\mathrm{Li}}\right)$, which is measured under a potential gradient, is reported to range between $1.8 \times$ $10^{-14} \mathrm{~cm}^{2} / \mathrm{s}$ (Ref. 28) and $7.6 \times 10^{-11} \mathrm{~cm}^{2} / \mathrm{s},{ }^{29}$ depending on the measurement technique. It is therefore highly required to estimate reliable $D_{\mathrm{Li}}$ of $\mathrm{LiFePO}_{4}$ experimentally.

Neutron experiments also revealed that $\mathrm{LiFePO}_{4}$ enters into a collinear AF ground state below $T_{\mathrm{N}} \cdot{ }^{21,23,30}$ The magnetic moments of the four Fe ions present in the unit cell are parallel/antiparallel to the $b$ axis with the ordered moment ( $\left.\mu_{\text {ord }}\right) 4.19 \mu_{\mathrm{B}}$ at $2 \mathrm{~K}$. Since such AF order generates a large internal magnetic field $\left(H_{\text {int }}\right)$ at each moun site, we can expect a clear muon-spin precession signal in a zero-field (ZF) $\mu^{+} \mathrm{SR}$ spectrum below $T_{\mathrm{N}}$. If there are multiple muon sites with different $H_{\text {int }}$ in the lattice, we could reconfirm the AF spin structure from the intensity ratio between multiple $H_{\text {int }}$, as in the case for $\mathrm{BaCoO}_{3},{ }^{31} \mathrm{Ba}_{2} \mathrm{CoO}_{4},{ }^{32}$ and $\mathrm{NaV}_{2} \mathrm{O}_{4} \cdot{ }^{33}$

Here, we report the low- $T$ magnetism and high- $T$ diffusive behavior of $\mathrm{LiFePO}_{4}$ investigated by $\mu^{+}$SR. Since $\mu^{+}$SR sensitively probes $H_{\text {int }}$ originated by both electron and nuclear, the change in $H_{\text {int }}$ with $T$ is clarified in a microscopic spatial scale.

\section{EXPERIMENT}

A powder sample of $\mathrm{LiFePO}_{4}$ was prepared by a solid-state reaction technique using reagent grade $\mathrm{Li}_{2} \mathrm{CO}_{3}$, $\mathrm{Fe}(\mathrm{II}) \mathrm{C}_{2} \mathrm{O}_{4} \cdot 2 \mathrm{H}_{2} \mathrm{O}$, and $\left(\mathrm{NH}_{4}\right)_{2} \mathrm{HPO}_{4}$ as starting materials. A stoichiometric mixture of the raw materials was thoroughly mixed by a conventional planetary milling apparatus, and then, the mixture was sintered at $700^{\circ} \mathrm{C}$ for $6 \mathrm{~h}$ in a purified argon gas flow. According to a powder $\mathrm{x}$-ray-diffraction (XRD) analysis, the sample was assigned as a single phase of an orthorhombic symmetry with space group Pnma.

In order to know the macroscopic magnetic properties of the sample, susceptibility $(\chi)$ was measured below $400 \mathrm{~K}$ under a
$H \leqslant 10 \mathrm{kOe}$ field with a superconducting quantum interference device (SQUID) magnetometer (MPMS, Quatum Design). The Weiss temperature $\left(\Theta_{\mathrm{CW}}\right)$ and effective magnetic moment $\left(\mu_{\text {eff }}\right)$ were determined from the $\chi(T)$ curve by fitting to a Curie-Weiss law, $\chi=C /\left(T-\Theta_{\mathrm{CW}}\right)$ and $C=\left(N / 3 k_{\mathrm{B}}\right) \mu_{\mathrm{eff}}^{2}$ in the $T$ range between 100 and $400 \mathrm{~K}$. Here, $N$ is the number density of Fe spins, $\mu_{\text {eff }}$ is the effective magnetic moment per $\mathrm{Fe}$, and $k_{\mathrm{B}}$ is Boltzmann's constant. As a result, we obtained $\Theta_{\mathrm{CW}}=-92.1 \pm 0.7 \mathrm{~K}$ and $\mu_{\mathrm{eff}}=5.58 \pm 0.01 \mu_{\mathrm{B}}$, respectively (see Fig. 1). These values are reproducible to those for polycrystalline samples in literature, ${ }^{20,21,23,25}$ and are consistent with the average values along the three directions for a single-crystal sample. ${ }^{22}$

The $\mu^{+}$SR spectra were measured at the surface muon beam lines using the LAMPF spectrometer of TRIUMF in Canada, the ARGUS spectrometer of ISIS/Riken-RAL in UK, and the D-OMEGA1 spectrometer of MUSE/MLF/J-PARC in Japan. In TRIUMF, the approximately 500-mg powder sample was placed in an envelope with $1 \mathrm{~cm} \times 1 \mathrm{~cm}$ area, which is made of Al-coated Mylar tape with $50 \mu \mathrm{m}$ thickness in order to minimize the signal from the envelope. And then, the envelope was attached to a low-background sample holder in a liquid-He flow-type cryostat in the $T$ range between 1.8 and $250 \mathrm{~K}$. In Riken-RAL and J-PARC, on the other hand, a 2-g powder sample was pressed into a disk with $27 \mathrm{~mm}$ diameter and $1 \mathrm{~mm}$ thickness, and packed into an Au O-ring sealed titanium cell. The window of the cell was made of a Kapton film with $50 \mu \mathrm{m}$ thickness. The cell was mounted onto the $\mathrm{Cu}$ plate of a liquid-He flow-type cryostat in the $T$ range between 10 and $500 \mathrm{~K}$. The experimental techniques are described in more detail elsewhere. ${ }^{8}$

\section{RESULTS}

\section{A. Low-temperature magnetic properties}

\section{Weak transverse field $\mu^{+} R$}

In order to study the microscopic magnetic nature of $\mathrm{LiFePO}_{4}$, we initially measured $\mu^{+} \mathrm{SR}$ spectra in a weak

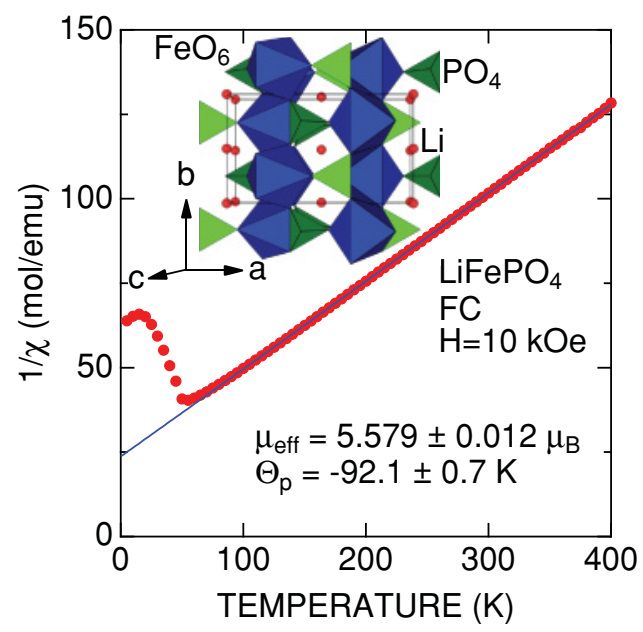

FIG. 1. (Color online) $T$ dependence of inverse susceptibility $(1 / \chi)$ for $\mathrm{LiFePO}_{4}$. The $\chi$ data were obtained in field cooling (FC) mode with $H=10 \mathrm{kOe}$. A solid line represents a linear fit in the $T$ range between 100 and $400 \mathrm{~K}$ using a Curie-Weiss formula. 
transverse field (wTF around 30 Oe). Here, "weak" means that the applied field is significantly less than any possible spontaneous internal fields $\left(H_{\text {int }}\right)$ in the ordered state. The WTF- $\mu^{+}$SR technique is sensitive to local magnetic order via the decrease in the amplitude (asymmetry) of the $\mu^{+}$ spin precession signal and the enhanced $\mu^{+}$spin relaxation. Figure 2(a) shows the variation of the wTF time spectra of $\mathrm{LiFePO}_{4}$ at different temperatures. When $T$ decreases below $55 \mathrm{~K}$, the oscillation amplitude due to WTF rapidly decreases below $51 \mathrm{~K}$, indicating the appearance of additional strong $H_{\text {int }}$. At $49 \mathrm{~K}$, the wTF oscillation eventually disappears and the spectrum consists of a fast relaxing signal in an early time domain and a slowly relaxing tail signal. Note that the initial amplitude of the wTF oscillation $\left(A_{0}\right)$ is below 0.2 even at $55 \mathrm{~K}$, although $A_{0} \sim 0.24$ for a Ag reference. This means that electron-induced magnetic moments are still fluctuating within a $\mu^{+}$SR time scale even above $T_{\mathrm{N}}$. The wTF- $\mu^{+} \mathrm{SR}$ spectrum was consequently fitted using a combination of a slowly relaxing precessing signal and two exponentially relaxing nonoscillatory signals. The first component is due to
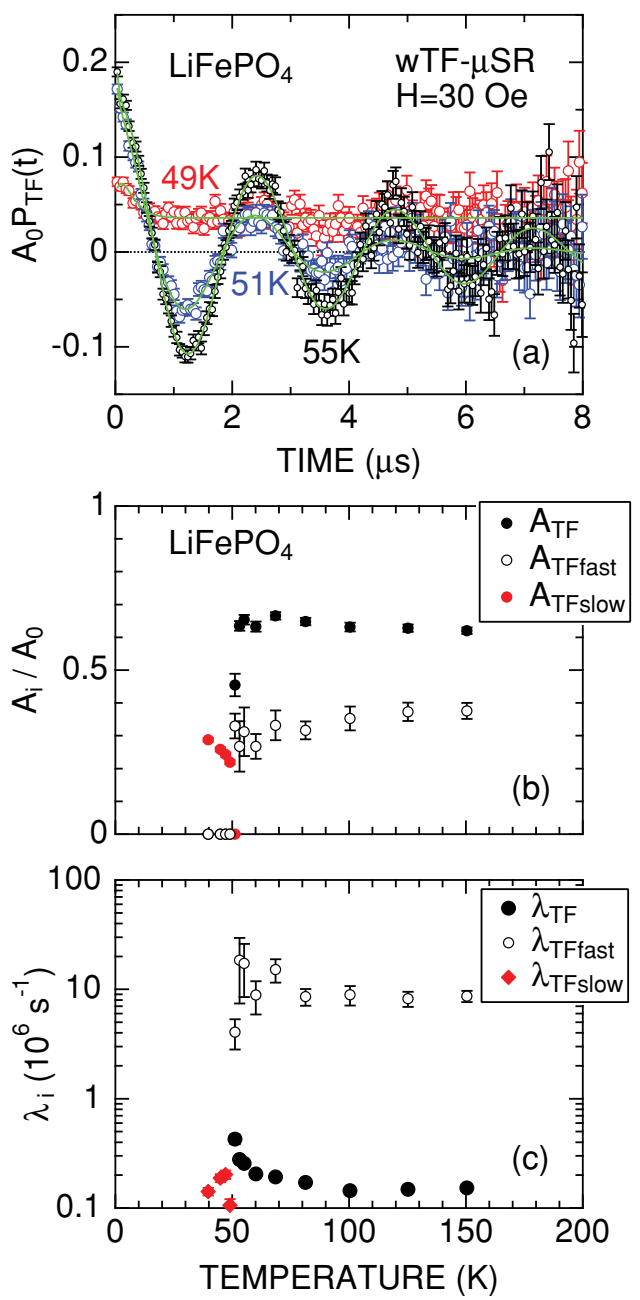

FIG. 2. (Color online) $T$ dependences of (a) the weak transverse field spectrum for $\mathrm{LiFePO}_{4}$ measured in TRIUMF, (b) normalized asymmetries $\left(A_{i} / A_{0}\right)$, and (c) relaxation rates $\left(\lambda_{i}\right)$. The data in (b) and (c) were obtained by fitting the wTF spectrum using Eq. (1). the externally applied magnetic field $(\mathrm{wTF}=30 \mathrm{Oe})$ and the second and third are due to $H_{\text {int }}$. More correctly, the third signal corresponds to the " $1 / 3$ tail" caused by the $H_{\text {int }}$ component parallel to the initial muon-spin polarization:

$$
\begin{aligned}
A_{0} P_{\mathrm{TF}}(t)= & A_{\mathrm{TF}} \cos \left(\omega_{\mathrm{TF}}^{\mu} t+\phi_{\mathrm{TF}}\right) \exp \left(-\lambda_{\mathrm{TF}} t\right) \\
& +A_{\mathrm{TFfast}} \exp \left(-\lambda_{\mathrm{TFfast}} t\right) \\
& +A_{\mathrm{TFslow}} \exp \left(-\lambda_{\mathrm{TFslow}} t\right),
\end{aligned}
$$

where $A_{0}$ is the initial ( $\left.t=0\right)$ asymmetry, $P_{\mathrm{TF}}(t)$ is the muon spin polarization function, $\omega_{\mathrm{TF}}^{\mu}$ is the muon Larmor frequency corresponding to the applied $\mathrm{wTF}, \phi_{\mathrm{TF}}$ is the initial phase of the precessing signal, $\lambda_{\mathrm{TF}}, \lambda_{\mathrm{TFfast}}$, and $\lambda_{\mathrm{TFslow}}$ are the exponential relaxation rates, and $A_{\mathrm{TF}}, A_{\mathrm{TFfast}}$, and $A_{\mathrm{TFs}}$ are the asymmetries of the three components of the $\mu^{+} \mathrm{SR}$ spectrum.

By plotting $A_{\mathrm{TF}}$ versus $T$ [see Fig. 2(b)], we can clearly see that the $\mathrm{LiFePO}_{4}$ sample has a bulk AF transition at $T_{\mathrm{N}}^{\mathrm{TF}}=$ $50.7 \mathrm{~K}$, where the normalized $A_{\mathrm{TF}}\left(=A_{\mathrm{TF}} / A_{0}=N_{A_{\mathrm{TF}}}\right)=0.5$. This is because the normalized $A_{\mathrm{TF}}$ corresponds to the volume fraction of paramagnetic (PM) phases in a sample. Interestingly, as $T$ decreases from $150 \mathrm{~K}, A_{\text {TFfast }}$ slightly decreases down to $T_{\mathrm{N}}$, and then, disappears at $T_{\mathrm{N}}$. Below $T_{\mathrm{N}}$, $A_{\text {TFslow }}$ appears instead and approaches to $1 / 3$ with further decreasing $T$. Here, we wish to mention the appearance of another fast relaxing component below $T_{\mathrm{N}}$, which was clearly detected by zero-magnetic-field measurements, as explained in Sec. III A 2.

As $T$ decreases from $150 \mathrm{~K}$, the $\lambda_{\mathrm{TF}}(T)$ curve exhibits a critical behavior towards $T_{\mathrm{N}}$ [Fig. 2(c)], while such behavior is very weak for the $\lambda_{\text {TFfast }}(T)$ curve. The origin of the $A_{\text {TFfast }}$ signal will be discussed in Sec. IV B. On the other hand, $\lambda_{\text {TFslow }}$ decreases with decreasing $T$, as expected for the $1 / 3$ tail signal (see also Sec. III A 2).

\section{Zero-field $\mu^{+} S R$}

In order to further elucidate the nature of the magnetic phase, we measured the $\mu^{+} \mathrm{SR}$ spectra in zero magnetic field (ZF). The $\mathrm{ZF}-\mu^{+} \mathrm{SR}$ technique is uniquely sensitive to local magnetic [dis]order in samples exhibiting quasistatic paramagnetic moments. Figure 3(a) shows the $T$ dependence of the $\mathrm{ZF}$ time spectrum in an early time domain for $\mathrm{LiFePO}_{4}$ and the Fourier transform frequency spectrum of the $\mathrm{ZF}$ time spectrum at the lowest $T$ measured. The time spectrum shows a clear oscillation below $T_{\mathrm{N}}$, and the frequency spectrum indicates the existence of two oscillatory signals with $\sim 45 \mathrm{MHz}$ and $\sim 90 \mathrm{MHz}$ at $1.8 \mathrm{~K}$. In addition, there is a very fast relaxing signal in the initial $0.01-0.02 \mu \mathrm{s}$ in the time spectra.

In fact, the ZF spectra were well fitted by a combination of two exponentially relaxing cosine oscillations for the static internal field and two exponentially relaxing nonoscillatory signals,

$$
\begin{aligned}
A_{0} P_{\mathrm{ZF}}(t)= & A_{\mathrm{AF} 1} \cos \left(\omega_{\mathrm{AF} 1}^{\mu} t+\phi_{\mathrm{AF} 1}\right) \exp \left(-\lambda_{\mathrm{AF} 1} t\right) \\
& +A_{\mathrm{AF} 2} \cos \left(\omega_{\mathrm{AF} 2}^{\mu} t+\phi_{\mathrm{AF} 2}\right) \exp \left(-\lambda_{\mathrm{AF} 2} t\right) \\
& +A_{\text {fast }} \exp \left(-\lambda_{\text {fast }} t\right)+A_{\text {slow }} \exp \left(-\lambda_{\text {slow }} t\right),
\end{aligned}
$$

where $A_{0}$ is the initial asymmetry, $A_{\mathrm{AF} 1}, A_{\mathrm{AF} 2}, A_{\text {fast }}$, and $A_{\text {slow }}$ are the asymmetries associated with the four signals. 


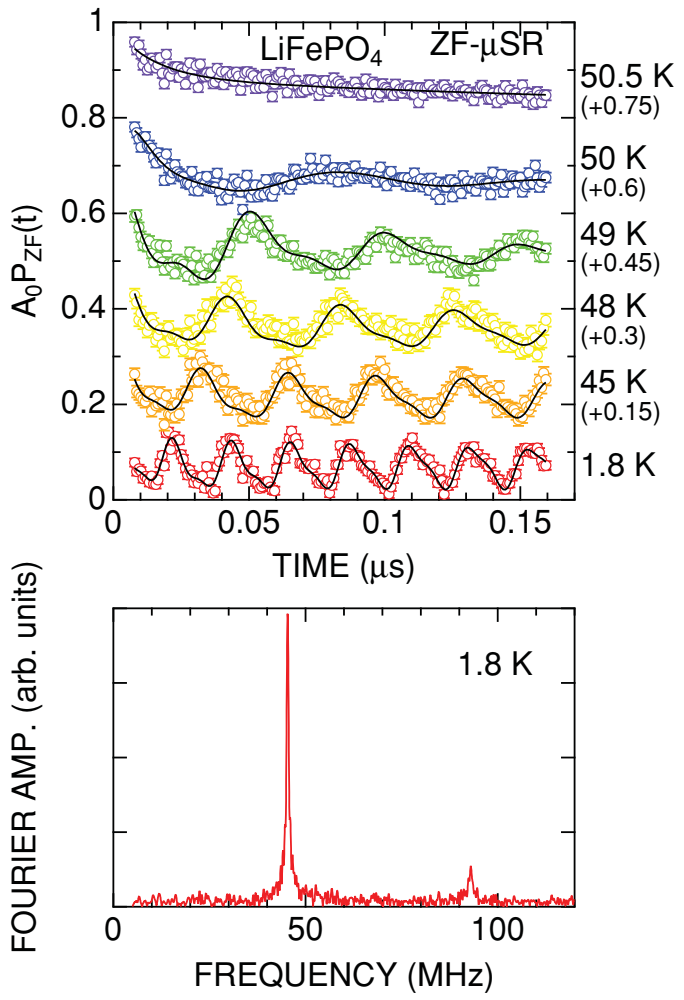

FIG. 3. (Color online) (a) $T$ dependence of the ZF- $\mu^{+}$SR time spectra and (b) the Fourier transform of the ZF spectrum at the lowest $T$ measured. In (a), each spectrum is offset by 0.15 for clarity of display. These spectra were obtained in TRIUMF.

$\omega_{\mathrm{AFi}}^{\mu}\left(\equiv 2 \pi f_{\mathrm{AFi}}\right)$ is the muon Larmor frequency corresponding to the static internal AF field, $\phi_{\mathrm{AFi}}$ is the initial phase of the oscillatory signal, $\lambda_{\mathrm{AFi}}, \lambda_{\text {fast }}$, and $\lambda_{\text {slow }}$ are the exponential relaxation rates of the four signals.

Figures 4(a)-4(f) show the $T$ dependences of the ZF- $\mu^{+}$SR parameters. The two $f_{\mathrm{AFi}}(T)$ curves [Fig. 4(a)] exhibit an order-parameter-like $T$ dependence. That is, as $T$ decreases from $T_{\mathrm{N}}$, both $f_{\mathrm{AF} 1}$ and $f_{\mathrm{AF} 2}$ increase monotonically down to the lowest $T$ measured with decreasing the slope $\left(d f_{i} / d T\right)$. Furthermore, the $T$ dependence of $f_{\mathrm{AF} 1}$ is found to be very similar to that of $f_{\mathrm{AF} 2}$. In fact, the ratio $f_{\mathrm{AF} 1} / f_{\mathrm{AF} 2}$ is 2.1 below $T_{\mathrm{N}}$, as seen in Fig. 4(f). If we employ a mean-field theory for the $f_{\mathrm{AFi}}(T)$ curves in the $T$ range between 20 and $50.1 \mathrm{~K}$, i.e., $f_{\mathrm{AFi}} / f_{\mathrm{AFi}}(0 \mathrm{~K})=\left[\left(T_{\mathrm{N}}-T\right) / T_{\mathrm{N}}\right]^{\beta}$, the critical exponent $(\beta)$ is estimated as $0.244(2)$ and $T_{\mathrm{N}}^{\mathrm{ZF}}$ as $50.20(2) \mathrm{K}$. These values are consistent with those obtained by neutron measurements in the same $T$ range, $\left[\beta^{\text {neutron }}=0.27(3)\right.$ and $\left.T_{\mathrm{N}}^{\text {neutron }}=50.0(5) \mathrm{K}\right] .^{30}$

The four asymmetries are also $T$ independent below $\sim 40 \mathrm{~K}$ [Fig. 4(b)]. In particular, since $A_{\text {slow }} \sim 1 / 3, A_{\text {slow }}$ is most likely as a " $1 / 3$ tail" signal caused by the field component parallel to the initial muon-spin polarization. It should be noted that the $A_{\text {fast }}$ signal is the largest among the four asymmetries. Besides the $1 / 3$ tail signal, the usage of the three independent signals in Eq. (2) does not mean the existence of three different magnetic phases in the sample but indicate the presence of three magnetically different muon sites in the $\mathrm{LiFePO}_{4}$ lattice, because all the signals disappear above $T_{\mathrm{N}}$. The assignment of the three
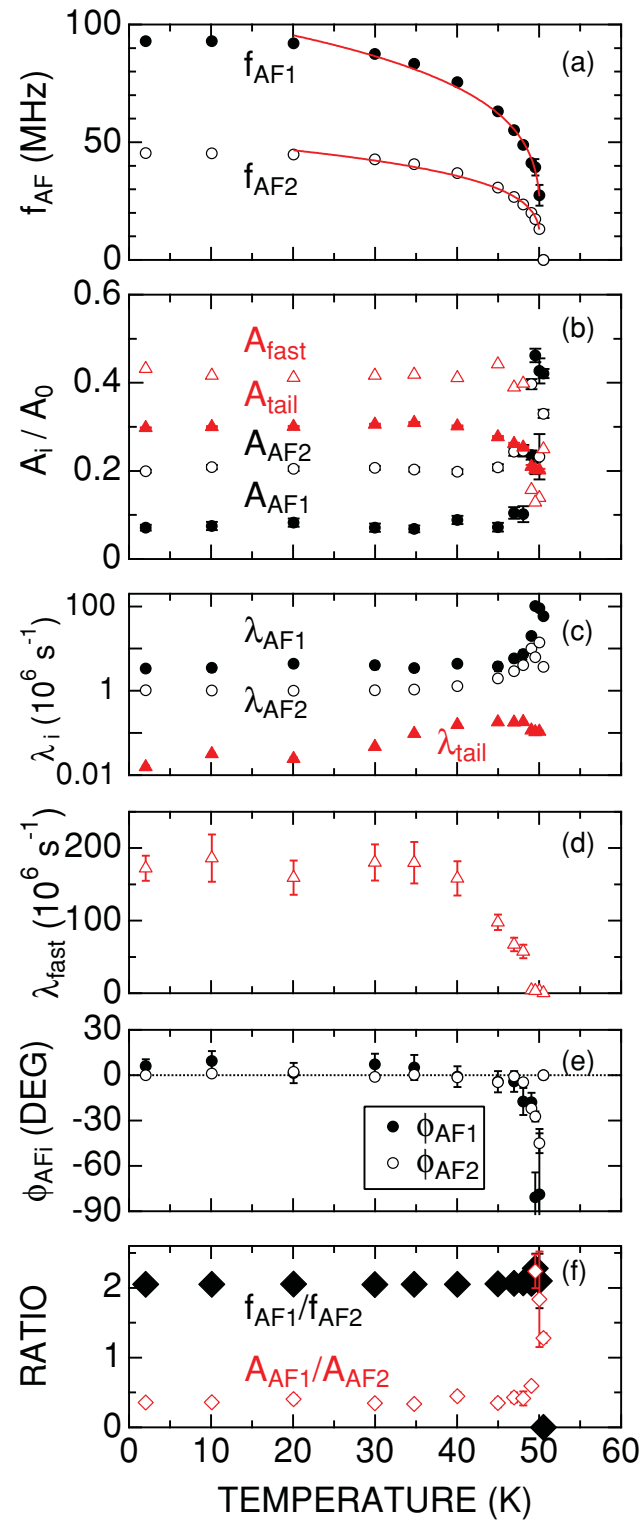

FIG. 4. (Color online) $T$ dependences of $\mu^{+}$SR parameters for the $\mathrm{LiFePO}_{4}$ sample; (a) muon-spin precession frequencies $\left(f_{\mathrm{AFi}}=\right.$ $\left.\omega_{\mathrm{AFi}}^{\mu} / 2 \pi\right),(\mathrm{b})$ the normalized asymmetries $\left(A_{i} / A_{0}\right),(\mathrm{c})$ the relaxation rates $\left(\lambda_{\mathrm{AFi}}\right)$, (d) the relaxation rate of the $A_{\text {fast }} \operatorname{signal}\left(\lambda_{\text {fast }}\right)$, (e) the initial phases of the two oscillatory signals $\left(\phi_{\mathrm{AFi}}\right)$, and (f) the ratios between $f_{\mathrm{AF} 1}$ and $f_{\mathrm{AF} 2}$ and $A_{\mathrm{AF} 1}$ and $A_{\mathrm{AF} 2}$. The data were obtained by fitting the ZF spectra using Eq. (2). In (a), solid lines represent the fitting result at $T \geqslant 20 \mathrm{~K}$ using the equation $f_{\mathrm{AFi}} / f_{\mathrm{AFi}}(0 \mathrm{~K})=$ $\left[\left(T_{\mathrm{N}}-T\right) / T_{\mathrm{N}}\right]^{\beta}$.

signals to the muon sites will be discussed in Sec. IV A. Here, we wish to emphasize that such a situation is often observed for magnetic materials, depending on its crystallographic and magnetic spin structures. For instance, although there is crystallographically only one muon site in the AF insulating $\mathrm{LiCrO}_{2}$ lattice, there are 36 magnetically different muon sites in the $\mathrm{AF}$ phase. The $\mathrm{ZF}$ spectrum for the $\mathrm{AF}$ phase of $\mathrm{LiCrO}_{2}$ is well explained by the sum of the 36 independent oscillatory signals plus a $1 / 3$ tail signal. ${ }^{35}$ Furthermore, for an AF metallic compound, $\mathrm{NaV}_{2} \mathrm{O}_{4}$, there are crystallographically 
four muon sites in the lattice, resulting in the appearance of four oscillatory signals in the ZF spectrum below $T_{\mathrm{N}} \cdot{ }^{33,36}$ Multiple oscillatory signals are also observed in the $\mathrm{ZF}$ spectrum for the AF phase of $\mathrm{BaCoO}_{3}$ (Ref. 31) and $\mathrm{Ba}_{2} \mathrm{CoO}_{4}{ }^{32}$

Although the two relaxation rates $\lambda_{\mathrm{AF} 1}$ and $\lambda_{\mathrm{AF} 2}$ exhibit a maximum below the vicinity of $T_{\mathrm{N}}$, they decrease rapidly with decreasing $T$, and level off to a constant value below $\sim 40 \mathrm{~K}$ [Fig. 4(c)], below which $A_{\mathrm{AF} 1}$ and $A_{\mathrm{AF} 2}$ are also $T$ independent. On the other hand, $\lambda_{\text {slow }}$ decreases monotonically with decreasing $T$, and approaches to 0 with $T \rightarrow 0$. Furthermore, since $\lambda_{\text {slow }} \ll \lambda_{\mathrm{AF} 2}, A_{\text {slow }}$ is reasonably assigned as a $1 / 3$ tail signal. Interestingly, the $\lambda_{\text {fast }}(T)$ curve shows a similar $T$ dependence to $f_{\mathrm{AF} 1}(T)$ and $f_{\mathrm{AF} 2}(T)$ [Figs. 4(a) and 4(d)], indicating that $A_{\text {fast }}$ comes from the fluctuating internal AF field. The origin of the $A_{\text {fast }}$ signal is discussed later.

Finally, except for a small temperature range in the vicinity of $T_{\mathrm{N}}$, both $\phi_{\mathrm{AF} 1}$ and $\phi_{\mathrm{AF} 2}$ are 0 [Fig. 4(e)]. This suggests a commensurate AF structure to the lattice period. We should note that all the $\mathrm{ZF}-\mu^{+} \mathrm{SR}$ parameters show a monotonic change in the $T$ range between $T_{\mathrm{N}}$ and $1.8 \mathrm{~K}$. The present results hence suggest the absence of an additional magnetic transition below $T_{\mathrm{N}}$, in contrast to the result of very recent Mössbauer measurements. ${ }^{34}$ The relationship between the AF spin structure proposed by neutron and the $\mu^{+}$SR parameters will be discussed in Sec. IV A.

\section{B. High-temperature diffusive behavior}

In the paramagnetic state above $T_{\mathrm{N}}$, the $\mathrm{ZF}-\mu^{+} \mathrm{SR}$ spectrum consists of a fast relaxation due to paramagnetic Fe moments $\left(H_{\text {int }}^{\mathrm{Fe}}\right)$ and a slow relaxation due to a nuclear magnetic field $\left(H_{\mathrm{int}}^{\mathrm{N}}\right.$ ) caused by ${ }^{6} \mathrm{Li},{ }^{7} \mathrm{Li}$, and ${ }^{31} \mathrm{P}$, as expected from the wTF measurements. Figure 5 shows the $\mathrm{ZF}$ and longitudinal field (LF) $\mu^{+}$SR spectrum obtained at (a) $100 \mathrm{~K}$, (b) $250 \mathrm{~K}$, and (c) $350 \mathrm{~K}$. Although the applied LF $(=10 \mathrm{Oe})$ clearly reduces the relaxation rate of the slow relaxation, i.e., decouples $H_{\mathrm{int}}^{\mathrm{N}}$, at $100 \mathrm{~K}$, such a "decoupling" effect is very weak at $250 \mathrm{~K}$, indicating the increase in a field fluctuation rate of $H_{\text {int }}^{\mathrm{N}}$ with $T$. Interestingly, the same LF apparently reduces the relaxation rate again at $350 \mathrm{~K}$. This means that $H_{\text {int }}^{\mathrm{N}}$ shows a static nature at $100 \mathrm{~K}$, but dynamic at $250 \mathrm{~K}$, and then becomes static again at $350 \mathrm{~K}$.

In order to know the change in $H_{\mathrm{int}}^{\mathrm{Fe}}$ and $H_{\mathrm{int}}^{\mathrm{N}}$ with $T$, the $\mathrm{ZF}$ and LF spectra were fitted simultaneously by a combination of an exponentially relaxation signal caused by $H_{\text {int }}^{\mathrm{Fe}}$, an exponentially relaxing dynamic Gaussian KT function $\left[G^{\text {DGKT }}\left(\Delta, v, t, H_{\mathrm{LF}}\right)\right]$ caused by a fluctuating $H_{\mathrm{int}}^{\mathrm{N}}$ due to $\mathrm{Fe}$ moments and Li diffusion, ${ }^{37}$ and an offset background (BG) signal from the fraction of muons stopped mainly in the sample cell, which is made of high-purity titanium,

$$
\begin{aligned}
A_{0} P_{\mathrm{LF}}(t)= & A_{\mathrm{F}} \exp \left(-\lambda_{\mathrm{F}} t\right) \\
& +A_{\mathrm{KT}} G^{\mathrm{DGKT}}\left(\Delta, \nu, t, H_{\mathrm{LF}}\right) \exp \left(-\lambda_{\mathrm{KT}} t\right)+A_{\mathrm{BG}},
\end{aligned}
$$

where $A_{0}$ is the initial $(t=0)$ asymmetry, $A_{\mathrm{F}}, A_{\mathrm{KT}}$, and $A_{\mathrm{BG}}$ are the asymmetries associated with the three signals. $\lambda_{\mathrm{F}}$ and $\lambda_{\mathrm{KT}}$ are the exponential relaxation rates, $\Delta$ is the static width of the local-field distribution at the disordered sites, and $v$ is the field fluctuation rate. When $v=0$ and
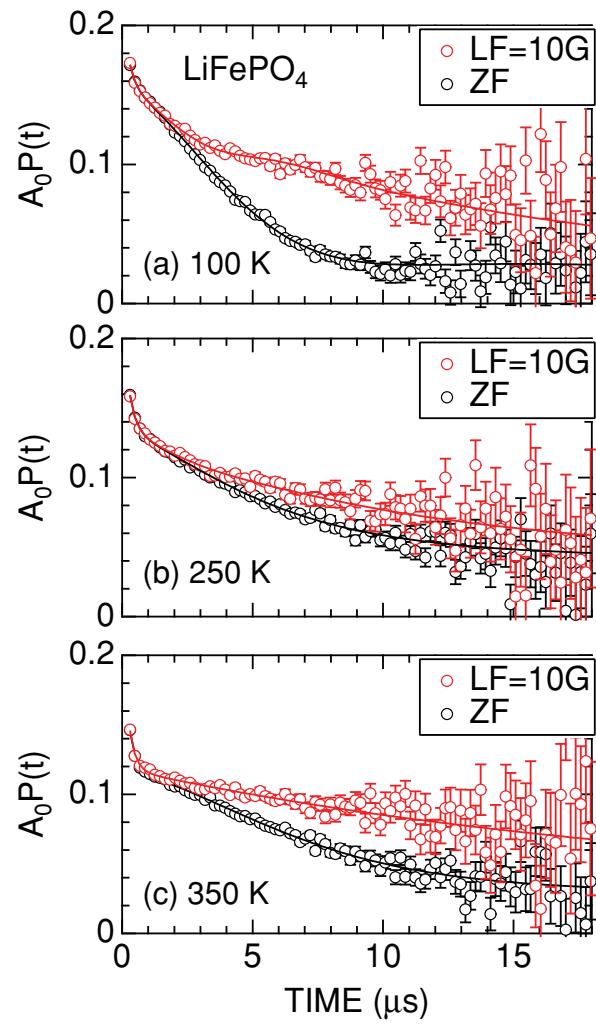

FIG. 5. (Color online) ZF- and LF- $\mu^{+}$SR spectra for $\mathrm{LiFePO}_{4}$ obtained at (a) $100 \mathrm{~K}$, (b) $250 \mathrm{~K}$, and (c) $350 \mathrm{~K}$. The magnitude of LF was 10 Oe. Solid lines represent the fit result using Eq. (3). These spectra were obtained in ISIS/RAL.

$H_{\mathrm{LF}}=0, G^{\mathrm{DGKT}}\left(t, \Delta, v, H_{\mathrm{LF}}\right)$ is the static Gaussian $\mathrm{KT}$ function $G_{z z}^{\mathrm{KT}}(t, \Delta)$ in $\mathrm{ZF}$. Equation (3) suggests that there are two different muon sites, as in the case for the low- $T$ results, for which there are two oscillatory signals and one fast relaxing signal, together with the $1 / 3$ tail signal. Correctly speaking, the $A_{\mathrm{F}}$ signal should be given by $A_{\mathrm{F}} G^{\mathrm{DGKT}}\left(\Delta, v, t, H_{\mathrm{LF}}\right) \exp \left(-\lambda_{\mathrm{F}} t\right)$, as well as the second term of Eq. (3). However, since $\lambda_{\mathrm{F}}$ is very large compared with $\Delta$ and $v$, as demonstrated later, $\exp \left(-\lambda_{\mathrm{F}} t\right)$ is predominant for the $A_{\mathrm{F}}$ signal. We thus used $A_{\mathrm{F}} \exp \left(-\lambda_{\mathrm{F}} t\right)$ instead. The assignment of the two signals to the muon sites will be discussed in Secs. IV A and IV B. Moreover, the $A_{\mathrm{BG}}$ term appears in Eq. (3) but does not in Eq. (2), because the sample cell was used only for high- $T \mu^{+}$SR measurements, as described in Sec. II.

At first, we fitted all the ZF and LF spectra using a common $A_{\mathrm{BG}}$ in the whole $T$ range and common, i.e., $H_{\mathrm{LF}}$ independent, $\Delta$ and $v$ at each $T$ in Eq. (3). Then, since both $\lambda_{\text {fast }}$ and $\lambda_{\mathrm{KT}}$ were found to be approximately $T$ independent, we finally used common $\lambda_{\mathrm{F}}$ and $\lambda_{\mathrm{KT}}$ for fitting the ZF and LF spectra. The obtained values are $A_{\mathrm{BG}}=0.0247(1), \lambda_{\mathrm{F}}=4.03(5) \times$ $10^{6} \mathrm{~s}^{-1}$, and $\lambda_{\mathrm{KT}}=0.0571(8) \times 10^{6} \mathrm{~s}^{-1}$.

Figure 6 shows the $T$ dependences of $\mu^{+}$SR parameters obtained by such a global fitting. As $T$ increases from $100 \mathrm{~K}, \Delta$ is almost $T$ independent until $200 \mathrm{~K}$, and decreases slightly with $T$, then finally levels off to a constant value $\left(\sim 0.1 \times 10^{-6} \mathrm{~s}^{-1}\right)$ above $\sim 300 \mathrm{~K}$. On the other hand, $v$ starts to increase above around $150 \mathrm{~K}$ with increasing the slope $(d v / d T)$, reaches a maximum 


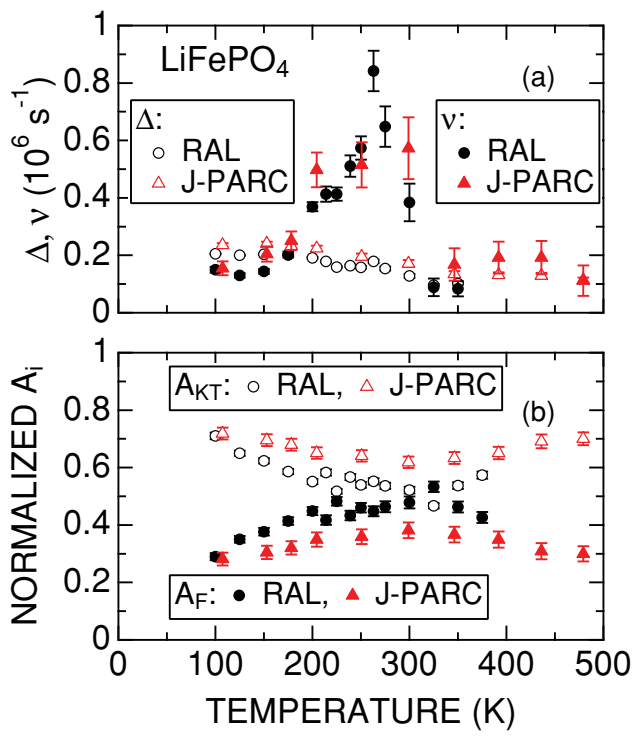

FIG. 6. (Color online) $T$ dependences of (a) $\Delta$ and $v$ and (b) normalized $A_{\mathrm{KT}}$ and $A_{\mathrm{F}}\left[A_{\mathrm{KT}} /\left(A_{\mathrm{KT}}+A_{\mathrm{F}}\right)\right.$ and $\left.A_{\mathrm{F}} /\left(A_{\mathrm{KT}}+A_{\mathrm{F}}\right)\right]$ for $\mathrm{LiFePO}_{4}$. Circles represent the data obtained in ISIS/RAL and triangles represent the data obtained in J-PARC. Each data was obtained by global-fitting the ZF and LF spectra using Eq. (3).

at $260 \mathrm{~K}$, and then decreases with further increasing $T$. Then, $v$ also becomes $T$ independent at $T$ above $325 \mathrm{~K}$.

It should be noted that there is no structural change around $260 \mathrm{~K}$, according to neutron-diffraction measurements. ${ }^{23}$ Moreover, detailed structural analyses of the XRD data, which were obtained in a synchrotron radiation $\mathrm{X}$-ray source (SPring-8), revealed a monotonic $T$ dependence of both the $\mathrm{Fe}-\mathrm{O}$ bond length and the $\mathrm{Fe}-\mathrm{O}-\mathrm{Fe}$ angle in the $T$ range between 100 and $300 \mathrm{~K}$ (see the Appendix). Then, if muons start to diffuse above $150 \mathrm{~K}$ and such diffusion is responsible for the $T$ dependence of $v$, it is very difficult to explain the static nature above $325 \mathrm{~K}$.

Therefore the most reasonable scenario is that the $\mathrm{Li}^{+}$ions start to diffuse above $150 \mathrm{~K}$ and their diffusion rate increases with $T$. Above $260 \mathrm{~K}$, since $v$ becomes rather large compared with $\Delta$, such diffusion is too fast to be visible by $\mu^{+} \mathrm{SR}$. As a result, $v$ decreases with $T$ above $260 \mathrm{~K}$, and finally, $v(\Delta)$ corresponds to the nuclear field fluctuation rate (nuclear field distribution width) mainly by ${ }^{31} \mathrm{P}$. Such $v$ and $\Delta$ are naturally static, because the $\mathrm{P}$ ions form the $\mathrm{PO}_{4}$ tetrahedra in the lattice.
The diffusive behavior detected by $\mu^{+} \mathrm{SR}$ will be discussed in detail in Sec. IV B.

The two asymmetries are found to vary with $T$, particularly at around $300 \mathrm{~K}$. That is, the $A_{\mathrm{KT}}(T)$ curve exhibits a broad minimum, but the $A_{\mathrm{F}}(T)$ curve exhibits a broad maximum, indicating the change in the occupancy of muon sites with $T$. This is because, since the $\mathrm{Li}^{+}$ions are diffusing, the distribution of electrostatic potential in the lattice is naturally altered by $\mathrm{Li}^{+}$diffusion. As a result, the stability of each muon site is thought to depend on $T$.

\section{DISCUSSION}

\section{A. Low-T AF phase}

Based on electrostatic potential calculations using a pointcharge model, there are four different muon sites at the vicinity of the $\mathrm{O}^{2-}$ ions in the $\mathrm{LiFePO}_{4}$ lattice. More correctly, we assumed that each $\mu$ is bound to the nearest $\mathrm{O}^{2-}$ ion with a typical bond length in oxides, namely, $d_{\mu-\mathrm{O}}=1 \AA$. $^{8}$ Then, we calculated the dipole field at each site using a collinear AF structure, which is proposed by a neutron experiment. ${ }^{23,30} \mathrm{As}$ a result, we obtained an internal $\mathrm{AF}$ field $\left(H_{\mathrm{AF}}\right)$ at each muon site, as listed in Table I. Since the AF ordered moment $\left(\mu_{\mathrm{ord}}^{\mathrm{ND}}\right)$ is reported as $4.19(5) \mu_{\mathrm{B}}$ at $2 \mathrm{~K},{ }^{23}$ one can expect the muon spin rotation signals with the precession frequency, 114, 70, 103 , and $103 \mathrm{MHz}$ in the $\mathrm{ZF}-\mu^{+} \mathrm{SR}$ spectrum. However, as seen in Fig. 4(a), $f_{\mathrm{AF} 1}=93 \mathrm{MHz}$ and $f_{\mathrm{AF} 2}=45 \mathrm{MHz}$ at $2 \mathrm{~K}$. This suggests that the muons responsible for the $A_{\mathrm{AF} 2}$ signal locate at the $\mu 12$ site, while those responsible for the $A_{\mathrm{AF} 1}$ signal must locate at one of the remaining three sites, i.e., the $\mu 11, \mu 21$, or $\mu 31$ site with $\mu_{\text {ord }}^{\mu \mathrm{SR}} \sim 2.9 \mu_{\mathrm{B}}$. The discrepancy between $\mu_{\text {ord }}^{\mu \mathrm{SR}}$ and $\mu_{\mathrm{ord}}^{\mathrm{ND}}$ is probably due to a different time window between the two techniques.

Next, we will discuss the origin of the $A_{\text {fast }}$ signal. Note that the $\lambda_{\text {fast }}(T)$ curve is similar to the $f_{\mathrm{AF} 1}(T)$ and $f_{\mathrm{AF} 2}(T)$ curve, but $\lambda_{\text {fast }}=180 \times 10^{6} \mathrm{~s}^{-1}$ at $2 \mathrm{~K}$. The magnitude of $H_{\text {int }}$ at the muon site(s) responsible for the $A_{\text {fast }}$ signal is hence likely to range below $f_{\text {fast }}=29 \mathrm{MHz}=\lambda_{\text {fast }} / 2 \pi\left(H_{\text {int }}=2100\right.$ Oe for $\mu^{+}$), although it is difficult to estimate $H_{\text {int }}$ for a nonoscillatory signal. This rough estimate, however, excludes the possibility of muonium formation in $\mathrm{LiFePO}_{4}$, because the gyromagnetic ratio of muonium in the triplet state $\left(\gamma_{\mathrm{Mu}} / 2 \pi\right)$ is $1.40 \mathrm{MHz} / \mathrm{Oe}$. As a result, the internal filed at the muonium site would be $21 \mathrm{Oe}$, which is too small for the AF ordered state and too large for the nuclear magnetic field (see Table I). Therefore we

TABLE I. Possible muon sites $\left(\mu_{n m}\right)$, which locate $1 \AA$ away from $\mathrm{O}_{n}$, the internal AF field $\left(H_{\mathrm{AF}}\right)$ at $\mu_{n m}$, electrostatic potential $(E)$ at $\mu_{n m}$, and nuclear dipole field distribution width $(\Delta)$ for $\mathrm{LiFePO}_{4}$ determined by electrostatic potential calculations and dipole field calculations. $\Delta \mathrm{FePO}_{4}$ is $\Delta$ without Li nuclear magnetic moments. The calculations were performed by a computer program DIPELEC (Ref. 38), which uses a point-charge model.

\begin{tabular}{|c|c|c|c|c|c|c|c|c|}
\hline Site & $\begin{array}{l}\text { Nearest O site } \\
\quad(x, y, z)\end{array}$ & $(x, y, z)$ & $\begin{array}{c}d_{\mu-\mathrm{Fe}} \\
(\AA)\end{array}$ & $\begin{array}{c}H_{\mathrm{AF}} \\
\left(\mathrm{Oe} / \mu_{\mathrm{B}}\right)\end{array}$ & $\begin{array}{c}f_{\mathrm{AF}} \\
\left(\mathrm{MHz} / \mu_{\mathrm{B}}\right)\end{array}$ & $\begin{array}{c}E \\
(\mathrm{eV})\end{array}$ & $\begin{array}{c}\Delta \\
\left(\times 10^{6} \mathrm{~s}^{-1}\right)\end{array}$ & $\begin{array}{c}\Delta^{\mathrm{FePO}_{4}} \\
\left(\times 10^{6} \mathrm{~s}^{-1}\right)\end{array}$ \\
\hline$\mu 11$ & O1 $(0.0971,0.2500,0.7428)$ & $(0.1225,0.3772,0.8679)$ & 1.885 & 1991 & 26.99 & -9.214 & 0.372 & 0.076 \\
\hline$\mu 12$ & O1 $(0.0971,0.2500,0.7428)$ & $(0.0416,0.2500,0.9172)$ & 2.501 & 1238 & 16.78 & -9.119 & 0.490 & 0.076 \\
\hline$\mu 21$ & $\mathrm{O} 2(0.4573,0.2500,0.2067)$ & $(0.3901,0.2500,0.3599)$ & 2.129 & 1803 & 24.44 & -11.176 & 0.265 & 0.061 \\
\hline$\mu 31$ & O3 $(0.8340,0.9536,0.7149)$ & $(0.8146,0.0404,0.8914)$ & 2.154 & 1800 & 24.39 & -10.788 & 0.202 & 0.065 \\
\hline
\end{tabular}




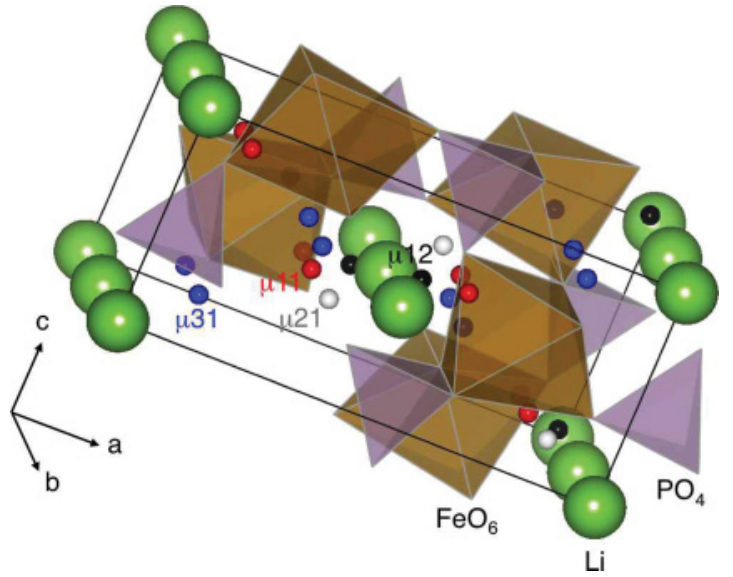

FIG. 7. (Color online) Possible muon sites $(\mu 11, \mu 12, \mu 21$, and $\mu 31)$ predicted by electrostatic potential calculations.

propose the following two scenarios for the origin of the $A_{\text {fast }}$ signal:

(1) Rapidly fluctuating Fe moments. Since $\mu_{\mathrm{ord}}^{\mu \mathrm{SR}}<\mu_{\mathrm{ord}}^{\mu \mathrm{ND}}$, Fe moments are still fluctuating even at $2 \mathrm{~K}$ from the viewpoint of $\mu^{+}$SR. Depending on the principal axis of the fluctuating Fe moments, the muons at a certain muon site would feel a dynamic field even below $T_{\mathrm{N}}$.

(2) Muon diffusion. As seen in Fig. 7, the distance between the nearest-neighboring $\mu 11$ sites is $1.106 \AA$. Since the two $\mu 11$ sites are symmetrical with respect to the $\mathrm{O} 1$ atom, muons at the $\mu 11$ site would jump between the neighboring sites, resulting in the fluctuating behavior.

Since $\lambda_{\text {fast }}$ decreases with $T$ below $T_{\mathrm{N}}$ [see Fig. 4(d)], the first scenario is more acceptable for the origin of the $A_{\text {fast }}$ signal. In this case, the muons responsible for the $A_{\text {fast }}$ signal are most likely to locate at the $\mu 11$ site due to the shortest distance to the nearest-neighboring $\mathrm{Fe}^{3+}\left(d_{\mu-\mathrm{Fe}}\right)$ among the three muon sites. The $\mu^{+} \mathrm{SR}$ signal is thus expected to be strongly affected by fluctuating Fe moments, leading to the exponentially relaxing signal.

\section{B. Li diffusive behavior}

The origin of the $A_{\mathrm{F}}$ signal is also thought to be fluctuating $\mathrm{Fe}$ moments in the paramagnetic phase, as for the origin of the $A_{\text {fast }}$ signal in the AF phase. Due to the insulating nature of $\mathrm{LiFePO}_{4}$ even above $T_{\mathrm{N}}, \lambda_{\mathrm{F}}$ is expected to obey a Curie-Weiss law, as well as $\chi$. However, since the change in $\chi$ above $100 \mathrm{~K}$ is not so drastic (Fig. 1), we could fit the $\mu^{+}$SR spectra using a common $\lambda_{\mathrm{F}}\left[=4.03(5) \times 10^{6} \mathrm{~s}^{-1}\right]$ until $500 \mathrm{~K}$. In addition, the fact that the $A_{\mathrm{F}}$ signal only affects the relaxation in an early time domain below $0.5 \mu \mathrm{s}$ (Fig. 5) makes it easy to fit the $\mu^{+}$SR spectra using a common $\lambda_{\mathrm{F}}$. According to our preliminary $\mu^{+} \mathrm{SR}$ work on the other phospho-olivines, $\mathrm{Li} M \mathrm{PO}_{4}(M=\mathrm{Mn}, \mathrm{Co}, \mathrm{Ni}),{ }^{39}$ the $A_{\mathrm{F}}$ signal is most likely to depend on $\mu_{\text {eff }}$ of $M$. That is, the ZF spectrum for $\mathrm{LiMnPO}_{4}$ with $\mu_{\text {eff }}=6.3 \mu_{\mathrm{B}}$ exhibits an exponentially relaxing behavior even at $300 \mathrm{~K}$, while that for $\mathrm{LiNiPO}_{4}$ with $\mu_{\text {eff }}=3.3 \mu_{\mathrm{B}}$ exhibits a KT behavior.

In order to explain the effect of the Fe moments on $\lambda_{\mathrm{F}}$, it is reasonable to assign that the muons at the $\mu 11$ site are responsible for the $A_{\mathrm{F}}$ signal due to the shortest $d_{\mu-\mathrm{Fe}}$. Indeed,

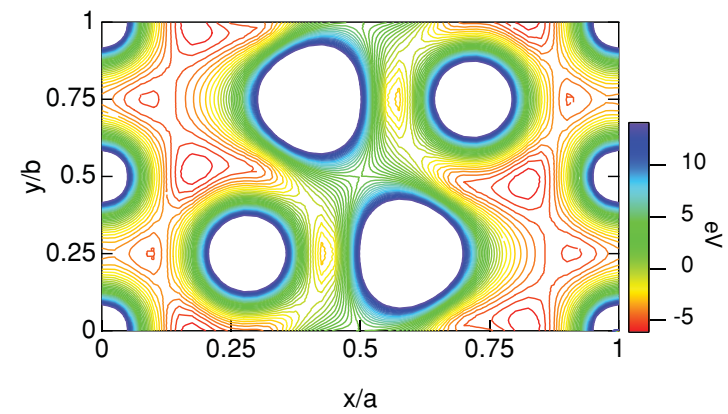

FIG. 8. (Color online) Electrostatic potential distribution in the $(x, y, 0)$ plane for $\mathrm{LiFePO}_{4}$. There are two potential minima around the regular Li sites, $(0,0,0)$ and $(0,0.5,0)$. That is, $(0.18,0.98$, $0)$ and $(0.09,0.75,0)$. The lattice constants are $a=10.3377 \AA$ and $b=6.0112 \AA$.

the normalized asymmetry for the $A_{\mathrm{F}} \operatorname{signal}(\sim 0.3$ at $100 \mathrm{~K})$ roughly agrees to that for the $A_{\text {fast }}$ signal $\left(0.41\right.$ below $\left.T_{\mathrm{N}}\right)$ in ZF measurements. Furthermore, the $A_{\text {TFfast }}$ signal in wTF measurements is naturally induced by the same origin to that for the $A_{\mathrm{F}}$ signal. In fact, the values of the normalized $A_{\mathrm{TFfast}}$ and $\lambda_{\text {TFfast }}$ are comparable to those of the normalized $A_{\mathrm{F}}$ and $\lambda_{\mathrm{F}}$.

The rest of the implanted muons, which locate at the $\mu 12$, $\mu 21$, and/or $\mu 31$ sites, however, feel the fluctuating field due to Li diffusion through a dynamic KT behavior, as in the case for $\mathrm{Li}_{x} \mathrm{CoO}_{2}$ and $\mathrm{LiNiO}_{2} \cdot{ }^{10,12}$ Such diffusion increases $v$ with increasing $T$ until $260 \mathrm{~K}$. At this $T$, since $v \sim 5 \Delta$, Eq. (3) becomes eventually equivalent to a simple exponential relaxation $[\exp (-\lambda t)]$. Thus the maximum in the $v(T)$ curve at $260 \mathrm{~K}$ indicates that the $\mathrm{Li}^{+}$diffusion is too fast for $\mu^{+} \mathrm{SR}$. In fact, $\Delta$ decreases slightly with $T$ above $200 \mathrm{~K}$ and levels off about a half of $\Delta(100 \mathrm{~K})$ above $\sim 350 \mathrm{~K}$, suggesting a motional narrowing of $\Delta$ due to Li diffusion. The dipole field calculations also supports this speculation, because $\Delta$ without the Li contribution $\left(\Delta^{\mathrm{FePO}_{4}}\right)$ ranges between $1 / 3 \Delta$ and $1 / 4 \Delta$ at the $\mu 11, \mu 21$, and $\mu 31$ sites (see Table I). Here, we wish to emphasize that the fastest limit of $v$ determined from a KT signal is not restricted by the magnitude of $v$ but by the ratio between $v$ and $\Delta$.

Finally, we attempt to evaluate a diffusion coefficient of $\mathrm{Li}^{+}$ ions $\left(D_{\mathrm{Li}}\right)$ using the present $\mu^{+} \mathrm{SR}$ result. Here, the regular $\mathrm{Li}$ site is fully occupied by $\mathrm{Li}$; we naturally consider only the jump to interstitial sites. The electrostatic potential calculations suggest two possible interstitial sites for Li diffusion, as seen in Fig. 8. Assuming that $v$ corresponds to the jump rate of the $\mathrm{Li}^{+}$ions between the neighboring sites, $D_{\mathrm{Li}}$ is given by ${ }^{40}$

$$
D_{\mathrm{Li}}=\sum_{i=1}^{n} \frac{1}{N_{i}} Z_{v, i} s_{i}^{2} v,
$$

where $N_{i}$ is the number of $\mathrm{Li}$ sites in the $i$ th path, $Z_{v, i}$ is the vacancy fraction, and $s_{i}$ is the jump distance. Therefore $n=2$, $N_{1}=2, s_{1}=1.86 \AA$, and $Z_{1}=1$ and $N_{2}=2, s_{2}=1.77 \AA$, and $Z_{2}=1$. Moreover, we assume that $\nu$ below $150 \mathrm{~K}$ is caused by the fluctuation of $\mathrm{Fe}$ moments. As a result, we have obtained the $T$ dependence of $D_{\mathrm{Li}}$ for $\mathrm{LiFePO}_{4}$ (Fig. 9). A linear relationship between $\ln \left(D_{\mathrm{Li}}\right)$ and $T^{-1}$ indicates a thermal activation process of $\mathrm{Li}$ diffusion, as expected. The magnitude of $D_{\mathrm{Li}}$ is calculated as $2.3 \times 10^{-10} \mathrm{~cm}^{2} / \mathrm{s}$ at $263 \mathrm{~K}$, and is estimated as $3.6 \times 10^{-10} \mathrm{~cm}^{2} / \mathrm{s}$ at $300 \mathrm{~K}$ by extrapolation of 


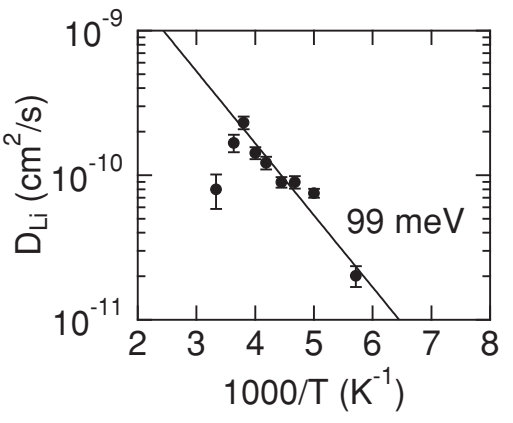

FIG. 9. The relationship between $D_{\mathrm{Li}}$ and $1 / T$. The straight line shows the thermally activated behavior discussed in the text.

the linear fit with the activation energy $\left(E_{a}\right) 99 \mathrm{meV}$. This value is about $1 / 5$ of $E_{a}$ along the $b$ direction $(540 \pm 50 \mathrm{meV})$ estimated from ac impedance measurements for single-crystal $\mathrm{LiFePO}_{4}{ }^{41}$ This is because $\mu^{+} \mathrm{SR}$ is specially sensitive to a short-range jump of $\mathrm{Li}^{+}$ions, while ac impedance senses the long-range Li diffusion. A very similar discrepancy between $E_{a}$ obtained by NMR and ac impedance is also reported for several materials. ${ }^{42}$

Since reliable $D_{\mathrm{Li}}$ for $\mathrm{LiFePO}_{4}$ estimated by other techniques are currently unavailable,$^{25,26}$ we compare the present result with the prediction by first-principles calculations. ${ }^{27}$ That is, $D_{\mathrm{Li}}$ is reported as $10^{-8} \mathrm{~cm}^{2} / \mathrm{s}$ for $\mathrm{Li}$ deficient $\mathrm{Li}_{7 / 8} \mathrm{FePO}_{4}$ at room temperature. The same calculations also provided that $E_{a}=270 \mathrm{meV}$. Note that these values are calculated for $\mathrm{Li}$ diffusion between a regular occupied site and regular vacant site. In other words, there is no prediction for stoichiometric $\mathrm{LiFePO}_{4}$. According to first-principles calculations for $\mathrm{Li}_{x} \mathrm{CoO}_{2}$, as $x$ increases from $7 / 8$ to $1, D_{\mathrm{Li}}$ is reported to decrease by $\sim 95 \%$. Therefore the discrepancy between the measured values for $\mathrm{LiFePO}_{4}$ and predicted values for $\mathrm{Li}_{7 / 8} \mathrm{FePO}_{4}$ would be acceptable. Also, it should be noted that $D_{\mathrm{Li}}$ for $\mathrm{LiFePO}_{4}$ is larger than those for $\mathrm{Li}_{x} \mathrm{CoO}_{2}$ and $\mathrm{LiNiO}_{2}$, despite the one-dimensional pathway for Li diffusion in $\mathrm{LiFePO}_{4}$. On the contrary, such high $D_{\mathrm{Li}}$ makes $\mathrm{LiFePO}_{4}$ useful as a cathode material for Li-ion batteries.

\section{SUMMARY}

We have investigated the low- $T$ magnetism and high- $T$ diffusive behavior of $\mathrm{LiFePO}_{4}$ by means of $\mu^{+} \mathrm{SR}$. The low $-T$ magnetic nature was well explained by a collinear AF model proposed by neutron scattering. Above $T_{\mathrm{N}}, \mu^{+} \mathrm{SR}$ detected $\mathrm{Li}^{+}$diffusion above $150 \mathrm{~K}$. Combining with the electrostatic potential calculations, a diffusion coefficient of $\mathrm{Li}$ ions were estimated as $3.6 \times 10^{-10} \mathrm{~cm}^{2} / \mathrm{s}$ at $300 \mathrm{~K}$.

\section{ACKNOWLEDGMENTS}

We thank the staff of J-PARC, ISIS, and TRIUMF for help with the $\mu^{+}$SR experiments. We also thank the staff of SPring-8 for help with the XRD experiment, which was approved by the Japan Synchrotron Radiation Research Institute (Proposal No. 2010B1806). Moreover, we thank K. Nishiyama of KEK for discussion. J.H.B. was supported at UBC by NSERC of Canada, and (through TRIUMF) by NRC of Canada, K.H.C. by NSERC of Canada and (through TRIUMF) by NRC of Canada. All images involving crystal structure were made with VESTA. ${ }^{43}$

\section{APPENDIX: STRUCTURAL ANALYSIS}

In order to study the $T$ dependences of the lattice parameters of $\mathrm{LiFePO}_{4}$, particularly at around $250 \mathrm{~K}$, we have measured powder XRD patterns as a function of $T$ at BL19B2 in SPring-8. The approximately 4-mg powder sample was filled into a glass capillary tube with $0.3 \mathrm{~mm}$ diameter. Then, the capillary tube was mounted on the goniometer, and the temperature of the sample/goniometer was controlled by a cold $\mathrm{N}_{2}$ gas flow. The XRD data were recorded on an imaging plate (IP) in the $T$ range between 100 and $450 \mathrm{~K}$. The wavelength of the incident synchrotron $\mathrm{x}$ ray was $0.6 \AA$. The obtained data were analyzed by a Rietvelt method $^{44}$ using the computer program RIETAN-FP. ${ }^{45}$

Figure 10 demonstrates the absence of a lattice anomaly in the whole $T$ range measured. This is very consistent with the result of neutron-scattering measurements. ${ }^{23}$ Therefore the maximum at $263 \mathrm{~K}$ in the $v(T)$ curve [Fig. 6(a)] is found to be not caused by a structural change in the lattice.
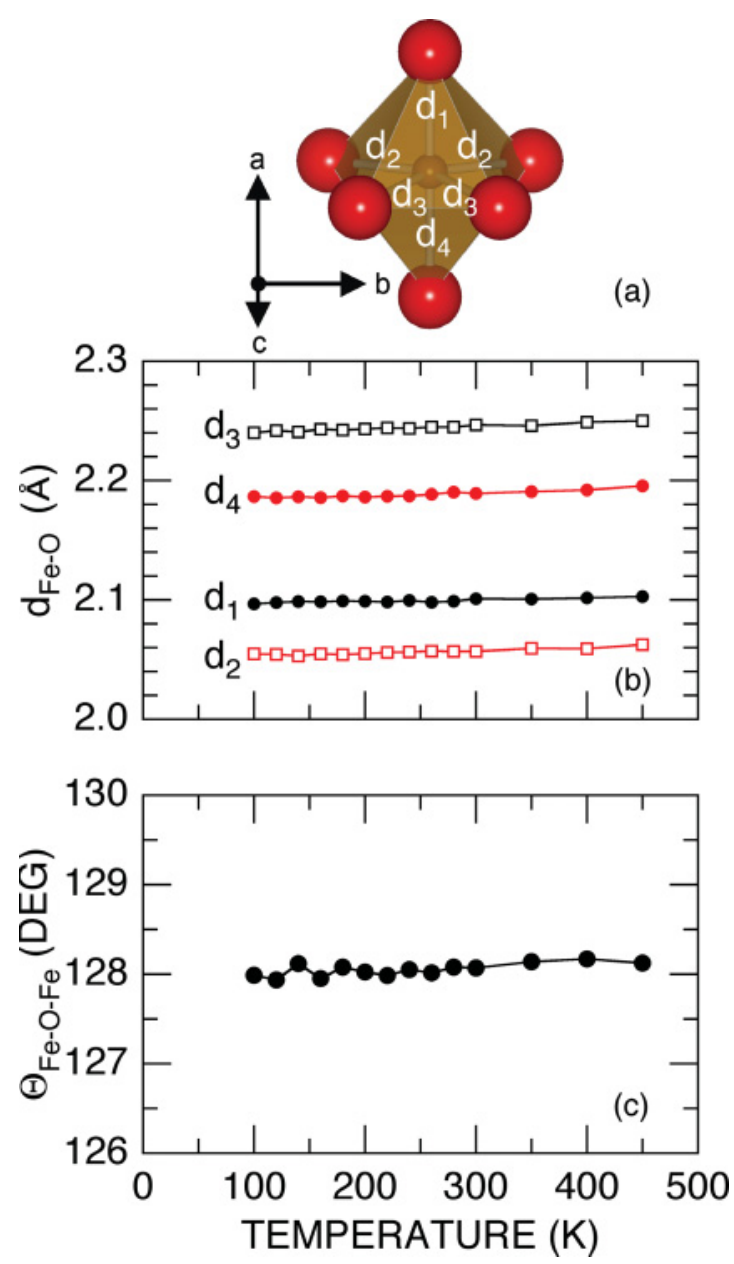

FIG. 10. (Color online) (a) The $\mathrm{FeO}_{6}$ octahedron and four bond lengths between $\mathrm{Fe}$ and $\mathrm{O}, d_{1}, d_{2}, d_{3}$, and $d_{4}$, in $\mathrm{LiFePO}_{4}$, (b) $T$ dependences of the four bond lengths, and (c) $T$ dependence of the angle between $\mathrm{Fe}-\mathrm{O}-\mathrm{Fe}$. 
*e0589@ mosk.tytlabs.co.jp

${ }^{\dagger}$ Present address: Research Center for Neutron Science and Technology, Comprehensive Research Organization for Science and Society (CROSS), 162-1 Shirakata, Tokai, Ibaraki 319-1106, Japan.

${ }_{\ddagger}^{\ddagger}$ Present address: Department of Material and Life Chemistry, Faculty of Engineering, Kanagawa University, Yokohama 221-8686, Japan.

${ }^{1}$ T. Ohzuku and R. Brodd, J. Power Sources 174, 449 (2007).

${ }^{2}$ K. Mizushima, P. C. Jones, P. J. Wiseman, and J. B. Goodenough, Mater. Res. Bull. 15, 783 (1980).

${ }^{3}$ I. Tomeno and M. Oguchi, J. Phys. Soc. Jpn. 67, 318 (1998).

${ }^{4}$ K. Nakamura, M. Yamamoto, K. Okamura, Y. Michihiro, I. Nakabayashi, and T. Kanashiro, Solid State Ionics 121, 301 (1999).

${ }^{5}$ K. Nakamura, H. Ohno, K. Okamura, Y. Michihiro, I. Nakabayashi, and T. Kanashiro, Solid State Ionics 135, 143 (2000).

${ }^{6}$ A. Van der Ven and G. Ceder, Electrochem. Solid-State Lett. 3, 301 (2000).

${ }^{7}$ R. S. Hayano, Y. J. Uemura, J. Imazato, N. Nishida, T. Yamazaki, and R. Kubo, Phys. Rev. B 20, 850 (1979).

${ }^{8}$ G. M. Kalvius, D. R. Noakes, and O. Hartmann, Handbook on the Physics and Chemistry of Rare Earths, edited by K. A. Gschneidner Jr., L. Eyring, and G. H. Lander (North-Holland, Amsterdam, 2001), Vol. 32, Chap. 206.

${ }^{9}$ J. Sugiyama, H. Nozaki, J. H. Brewer, E. J. Ansaldo, G. D. Morris, and C. Delmas, Phys. Rev. B 72, 144424 (2005).

${ }^{10}$ J. Sugiyama, K. Mukai, Y. Ikedo, H. Nozaki, M. Månsson, and I. Watanabe, Phys. Rev. Lett. 103, 147601 (2009).

${ }^{11}$ K. Nakamura, H. Ohno, K. Okamura, Y. Michihiro, T. Moriga, I. Nakabayashi, and T. Kanashiro, Solid State Ionics 177, 821 (2006).

${ }^{12}$ J. Sugiyama, Y. Ikedo, K. Mukai, H. Nozaki, M. Månsson, O. Ofer, M. Harada, K. Kamazawa, Y. Miyake, J. H. Brewer, E. J. Ansaldo, K. H. Chow, I. Watanabe, and T. Ohzuku, Phys. Rev. B 82, 224412 (2010).

${ }^{13}$ T. Ohzuku, A. Ueda, and M. Nagayama, J. Electrochem. Soc. 140, 1862 (1993).

${ }^{14}$ P. G. Bruce, Chem. Commun. 1817 (1997).

${ }^{15}$ A. Rougier, P. Gravereau, and C. Delmas, J. Electrochem. Soc. 143, 1168 (1996)

${ }^{16}$ S.-T. Myung, S. Komaba, N. Hirosaki, N. Kumagai, K. Arai, R. Kodama, and I. Nakai, J. Electrochem. Soc. 150, A1560 (2003).

${ }^{17}$ S. Komaba, C. Takei, T. Nakayama, A. Ogata, and N. Yabuuchi, Electrochem. Commun. 12, 355 (2010).

${ }^{18}$ A. K. Padhi, K. S. Nanjundaswamy, and J. B. Goodenough, J. Electrochem. Soc. 144, 1188 (1997).

${ }^{19}$ W.-J. Zhang, J. Power Sources 196, 2962 (2011), and references cited therein.

${ }^{20}$ C. M. Julien, A. Ait-Salah, A. Mauger, and F. Gendron, Ionics 12, 21 (2006)

${ }^{21}$ R. P. Santoro and R. E. Newnham, Acta Crystallogr. 22, 344 (1967).

${ }^{22}$ G. Liang, K. Park, J. Li, R. E. Benson, D. Vaknin, J. T. Markert, and M. C. Croft, Phys. Rev. B 77, 064414 (2008).
${ }^{23}$ G. Rousse, J. Rodriguez-Carvajal, S. Patoux, and C. Masquelier, Chem. Mater. 15, 4082 (2003).

${ }^{24}$ S. Nishimura, G. Kobayashi, K. Ohoyama, R. Kanno, M. Yashima, and A. Yamada, Nat. Mater. 7, 707 (2008).

${ }^{25}$ D. Arcon, A. Zorko, R. Dominko, and Z. Jagličič, J. Phys.: Condens. Matter 16, 5531 (2004).

${ }^{26}$ J. L. Dodd, Ph.D. thesis, California Institute of Technology, Pasadena, CA, 2007.

${ }^{27}$ D. Morgan, A. Van der Ven, and G. Ceder, Electrochem. Solid State Lett. 7, A30 (2004).

${ }^{28}$ P. P. Prosini, M. Lisi, D. Zane, and M. Pasquali, Solid State Ionics 148, 45 (2002).

${ }^{29}$ A. V. Churikov, A. V. Ivanishchev, I. A. Ivanishcheva, V. O. Sycheva, N. R. Khasanova, and E. V. Antipov, Electrochim. Acta 55, 2939 (2010).

${ }^{30}$ J. Li, V. O. Garlea, J. L. Zarestky, and D. Vaknin, Phys. Rev. B 73, 024410 (2006).

${ }^{31}$ H. Nozaki, M. Janoschek, B. Roessli, J. Sugiyama, L. Keller, J. H. Brewer, E. J. Ansaldo, G. D. Morris, T. Takami, and H. Ikuta, Phys. Rev. B 76, 014402 (2007).

${ }^{32}$ P. L. Russo, J. Sugiyama, J. H. Brewer, E. J. Ansaldo, S. L. Stubbs, K. H. Chow, R. Jin, H. Sha, and J. Zhang, Phys. Rev. B 80, 104421 (2009).

${ }^{33}$ O. Ofer, Y. Ikedo, T. Goko, M. Månsson, J. Sugiyama, E. J. Ansaldo, J. H. Brewer, K. H. Chow, and H. Sakurai, Phys. Rev. B 82, 094410 (2010).

${ }^{34}$ C. H. Rhee, I. K. Lee, S. J. Moon, S. J. Kim, and C. S. Kim, J. Korean Phys. Soc. 58, 472 (2011).

${ }^{35}$ J. Sugiyama, M. Månsson, Y. Ikedo, T. Goko, K. Mukai, D. Andreica, A. Amato, K. Ariyoshi, and T. Ohzuku, Phys. Rev. B 79, 184411 (2009).

${ }^{36}$ J. Sugiyama, Y. Ikedo, T. Goko, E. J. Ansaldo, J. H. Brewer, P. L. Russo, K. H. Chow, and H. Sakurai, Phys. Rev. B 78, 224406 (2008).

${ }^{37}$ T. Matsuzaki, K. Nishiyama, K. Nagamine, T. Yamazaki, M. Senba, J. M. Bailey, and J. H. Brewer, Phys. Lett. A 123, 91 (1989).

${ }^{38}$ K. M. Kojima, J. Yamanobe, H. Eisaki, S. Uchida, Y. Fudamoto, I. M. Gat, M. I. Larkin, A. Savici, Y. J. Uemura, P. P. Kyriakou, M. T. Rovers, and G. M. Luke, Phys. Rev. B 70, 094402 (2004).

${ }^{39}$ J. Sugiyama, J-PARC MLF experimental reports on 2010A0041 and 2010B0069.

${ }^{40}$ R. J. Borg and G. J. Dienes, in An Introduction to Solid State Diffusion (Academic, San Diego, 1988).

${ }^{41}$ J. Li, W. Yao, S. Martin, and D. Vaknin, Solid State Ionics 179, 2016 (2008).

${ }^{42}$ P. Heitjans and S. Indris, J. Phys.: Condens. Matter 15, R1257 (2003).

${ }^{43}$ K. Momma and F. Izumi, J. Appl. Crystallogr. 41, 653 (2008).

${ }^{44}$ H. M. Rietveld, J. Appl. Crystallogr. 2, 65 (1969).

${ }^{45}$ F. Izumi and K. Momma, Solid State Phenom. 130, 15 (2007). 